1 Text S1. Summary of FARMS methodology and findings on weed suppression strategies

2 for a tomato cropping system

3 annual extreme minimum temperature of -20 to $-15^{\circ} \mathrm{C}$ (USDA, 2012) and relatively warm

24 summers and cold winters. The production field where experiments were carried out is located

25 on a flood plain adjacent to the Connecticut River with open grass fields in the immediate

Here we synthesize student research findings from one of the five experiments carried out as part of the FARMS-based course at the Dartmouth Organic Farm on the effects of weed management in tomato cropping systems. To address the sustainability challenge of managing for weeds without the application of agrochemical input, students investigated three potential weed management practices for tomato cropping systems at the Dartmouth Organic Farm. The two weed management strategies investigated include cover cropping with clover and utilization of synthetic black plastic mulch. Plastic mulch is a widespread weed suppression method used for vegetable production and soil conservation as well as for regulating temperatures by absorbing sunlight and encouraging crop growth earlier in the growing season (Liebman and Gallandt, 1997). These weed strategies were compared to a control plot with no intentional weed management other than occasional manual weeding. The weed management treatments were applied to the cultivation of four indeterminate tomato cultivars (Sun Gold Cherry, Rose De Berne, Zebra, and Yellow Brandy) to observe any potential differences on the effects of various weed management strategies on different cultivars of the same crop. The overall research question for this experiment was: What are the effects of weed management treatments on crop vitality, weed abundance, soil quality, and crop quality in tomato cropping systems?

\section{Study Site}

Research was carried out at the Dartmouth Organic Farm in New Hampshire (Figure S1).

22 The study site is located in USDA plant hardiness zone 5A that is characterized by an average 
1 surroundings and nestled within a diverse temperate forest. The primary agroecological

2 management practice of the organic-certified production field is annual crop rotation divided into

3 six rotation sections of five botanical families (Solanaceae, Poaceae, Fabaceae, Brassicaeae, and

4 Cucurbitaceae) and one mixed family section. Each growing season, the different crop sections

5 are rotated in order to help reduce depletion of soil nutrients and help prevent outbreaks of

6 pathogens and pests. This annual crop rotation scheme provides a clear understanding of the

7 history of six sections of the production field at the Dartmouth Organic Farm. For example, the

8 previous year before the implementation of the FARMS experiment in the tomato plots (in

9 2016), plants in the Poaceae were planted in that location (in the summer of 2015). Prior to that,

10 plants in the Fabaceae were planted in that location (summer of 2014). This annual crop rotation

11 scheme has been implemented for over two decades at the Dartmouth Organic Farm.

\section{Experimental Procedures}

13 The sample size for each management treatment was twenty tomato plants that were

14 divided among the four tomato varieties for a sample size of five individual plants per

15 management strategy and tomato variety. The sample size was determined on the basis of power

16 analysis of data from previous similar agroecological experiments to determine if the results

17 have statistical significance. Due to time constraints of plant growth coupled with the course

18 duration, the experiment was set up prior to the course including planting of the tomatoes and

19 implementation of the weed management treatments. All experimental plots were manually

20 weeded once midpoint during the experiment.

21 In the first data collection period for the tomato experiment, students measured tomato

22 crop vitality parameters including height, number of leaves, number of branching stems, number

23 of fruits, leaf color, plant suppleness, and overall plant vitality approximately three weeks before 
1 the tomato plants were ready for harvest (the last three parameters were measured on a scale of

$20-5$; See Text S2). All tomato plants in the treatment plots were measured. These crop vitality

3 parameters were measured again right before the plants were ready for harvest. The number of

4 fruit were measured at three separate times because fruit are the major output around which

5 farmers make decisions regarding production and management.

6 Here we report on data from the second data collection of plant vitality measures that

7 preceded the harvest period. In the second data collection period for the tomato experiment,

8 students measured the prevalence and abundance of weeds in each of the treatment plots through

9 manual weeding, classification and sorting of weeds, and measuring dried biomass. Students

10 drew from classroom lectures, readings, stakeholder engagement, and other resources to identify

11 weeds and create hypotheses regarding what the presence and abundance of specific weeds

12 indicate regarding soil quality. Student measured soil parameters during the third data collection

13 period dedicated to the tomato experiment including soil temperature, soil moisture, $\mathrm{pH}$, nutrient

14 levels (including nitrogen, phosphorus, and potassium), and soil organic matter. In the final data

15 collection period dedicated to the tomato experiment, students collected tomato samples for

16 measurement of crop quality including sensory evaluation, brix levels, and total phenolic

17 concentration as previously reported (Ahmed et al., 2010).

18 Students collaborated in research teams to collect, analyze, and report data including the

19 preparation of research reports discussing the implication of findings for informing weed

20 management at the Dartmouth Organic Farm and more broadly for sustainable agriculture. JMP

21 (version 12.0 SAS Institute Inc., Cary, NC) was used for statistical analysis and creating graphs.

22 An Analysis of Variance (ANOVA) examined differences in means of the parameters measured 
1 while a multiple comparison using the LS Means Tukey HSD method was applied to examine if

2 individual measures significantly varied among treatments.

\section{$3 \quad$ Findings}

$4 \quad$ Crop Vitality. During the first data collection on plant vitality measures when the tomato

5 crops were young in their life cycle, no significant differences in the means of the various plant

6 vitality measures were found across the three weed suppression strategies for the overall tomato

7 experiment. However, during the second data collection on plant vitality measures that were

8 taken immediately before the tomato fruit were ready for harvest, significant differences were

9 observed in the means of the various plant vitality measures across the three weed suppression

10 strategies for the overall tomato experiment (Figure S2). Specifically, significant differences

11 were observed across the three treatments for plant height $(\mathrm{p}<0.0001)$, number of branching

12 stems $(\mathrm{p}<0.0102)$, and intensity of leaf color $(\mathrm{p}<0.0021)$. No significant differences were

13 observed across the three treatments for number of fruit $(\mathrm{p}<0.8005)$, number of leaves $(\mathrm{p}<$

14 0.9194), leaf strength $(\mathrm{p}<0.8603)$, and plant vitality $(\mathrm{p}<0.2724)$. The black plastic treatment

15 had the greatest plant height $(129.96 \mathrm{~cm})$ followed by the control $(116.04 \mathrm{~cm})$ and the clover

16 treatment (110.08). The pairwise comparison analysis showed that the plant height was

17 significantly more for the black plastic treatment while no significant differences were found

18 between the clover cover crop treatment and the control. The clover cover crop treatment had

19 significantly more branching stems (4.84) compared to both the black treatment (3.88) and the

20 control (3.68) with no significant differences found between the black plastic treatment and the

21 control. For the intensity of leaf color, a proxy measure for photosynthetic potential, the clover

22 cover crop treatment had significantly higher scores (3.76 on a $0-5$ scale) compared to the black 
1 plastic treatment (3.12) and control (2.96) with no significant differences found between the

2 latter.

3 On a cultivar basis, significant differences were seen in the means of several of the plant

4 vitality measures across the treatments during the second data collection period. For the Rose De

5 Berne cultivar, significant differences were observed in stem length during the second data

6 collection between the three treatments $(\mathrm{p}<0.0002)$ with the black plastic treatment having the

7 greatest stem length $(145.2 \mathrm{~cm})$ followed by the control $(122 \mathrm{~cm})$, and then the clover crop cover

8 treatment $(112.80 \mathrm{~cm})$. At the same time, significant differences were seen in the means for the

9 number of stems for the Rose De Berne cultivar during the second data collection between the

10 three treatments $(\mathrm{p}<0.0035)$ with the clover cover cropping treatment having the greatest

11 number of stems (mean of 6.8) followed by the black plastic mulch (3.6), and then the control

12 (3.4). No significant differences were seen in the means across treatments for the Rose De Berne

13 cultivar during the second data collection for number of fruit $(\mathrm{p}=0.3807)$, leaf intensity $(\mathrm{p}=$

14 0.6186), leaf strength $(p=0.5561)$, and overall plant vitality $(p=1.0)$. For Sungold Cherry, a

15 significant difference was seen in the means across treatments during the second data collection

16 for leaf intensity $(\mathrm{p}<0.0001)$, a proxy for plant health and photosynthetic potential, with the

17 clover cover cropping treatment (mean score of 4.4 on a scale of $0-5$ ) having the greatest score

18 followed by the black plastic mulch (3.7) and the control (2.9). No significant differences were

19 seen in the means across treatments for the Sungold Cherry cultivar during the second data

20 collection for stem length $(p=0.9166)$, number of fruit $(p=0.1699)$, number of stems $(p=$

21 0.7996), number of leaves $(\mathrm{p}=0.5584)$, leaf strength $(\mathrm{p}=0.5318)$, and overall plant vitality $(\mathrm{p}=$

22 0.1438). For Yellow Brandy, a significant difference was seen in the means across treatments

23 during the second data collection for stem length $(p=0.0103)$ and leaf strength $(p=0.0363)$. For 
1 stem length for Yellow Brandy, the black plastic treatment had the highest means $(124.20 \mathrm{~cm})$

2 followed by the control $(102.80 \mathrm{~cm})$ and the clover cover crop treatment $(95.60 \mathrm{~cm})$. For leaf

3 strength for Yellow Brandy, the control treatment had the highest means (score of 3.6 on a scale

4 from 0-5) followed by the black plastic treatment (score of 3.4) and the clover cover crop

5 treatment (2.40). No significant differences were seen in the means across treatments for the

6 Yellow Brandy cultivar during the second data collection for number of fruit $(\mathrm{p}=0.3794)$,

7 number of stems $(p=0.2397)$, number of leaves $(p=0.7391)$, and overall plant vitality $(p=$

8 0.1263). For Green Zebra, a significant difference was seen in the means across treatments

9 during the second data collection for stem length $(\mathrm{p}<0.0004)$, number of branching stems $(\mathrm{p}=$

10 0.0056), and leaf color intensity $(\mathrm{p}=0.0042)$. The black plastic treatment in the Green Zebra

11 plots had the highest stem length $(131.60 \mathrm{~cm})$ followed by the control $(107.40 \mathrm{~cm})$ and the

12 control $(98.60 \mathrm{~cm})$. The clover cover crop treatment in the Green Zebra plots had the greatest

13 number of branching stems (mean of 5.8) followed by the black plastic mulch (3.8) and the

14 control (3.6). Additionally, the clover cover crop treatment in the Green Zebra plots had the

15 highest leaf intensity scores (mean score of 4.0 on a $0-5$ scale) followed by the control (3.4) and

16 the black plastic treatment (2.4). No significant differences were seen in the means across

17 treatments for the Green Zebra cultivar during the second data collection for number of fruit $(\mathrm{p}=$

18 0.1548), number of leaves $(p=0.3236)$, leaf strength $(p=0.9009)$, and overall plant vitality $(p=$

$190.3000)$.

20 Weed Abundance. Findings showed a significant difference in the means of total weed

21 biomass across the three weed suppression strategies for the overall tomato experiment $(\mathrm{p}=$

22 0.0059; Figure S3). The pair wise comparison of the means of total weed biomass across the

23 three weed suppression strategies found that the clover cover crop treatment (285.58g) and the 
1 black plastic treatment (3.75g) had significantly less total weed biomass compared to the control

$2 \quad(1,063.45 \mathrm{~g})$ while no significant differences $(\mathrm{p}=0.29080)$ were seen between the clover cover

3 crop treatment and the black plastic. The Sungold Cherry plots had the greatest abundance of

4 weeds based on dried weed biomass among the four cultivars in the study, however, this

5 difference was not statistically significant $(\mathrm{p}=0.8494)$. Figure $\mathrm{S} 4$ shows how the abundance of

6 weed type varied with weed suppression treatment. For the control, the most abundant weeds

7 were lamb's quarter (Chenopodium album; 45.3\%; Figure S4) and pigweed (Amaranthus sp.;

$8 \quad 40.9 \%$ ) followed by grasses (9.7\%) and purslane (Portulaca oleracea; 4.1\%). For the clover

9 cover crop treatment, the most abundant weed was pigweed (57\%) followed by lamb's quarter

10 (20.1\%), grasses (11.9\%), and purslane (11\%). For the black plastic, the most abundant weed

11 was purslane (83.6\%) followed by grasses (6.9\%), lamb's quarter (5.4\%), and pigweed (4.1\%).

12 On the basis of the principle that the presence of specific weeds are indicators of soil conditions

13 (Pfeiffer, 1974), the presence of lamb's quarters, pigweed, and purslane in all three treatments

14 indicates the high fertility of the soil while the pigweed and purslane indicate that the soil was

15 previously cultivated. The presence of the lamb's quarters potentially indicates alkaline soils

16 (Rodale's Organic Life, 2012).

17 While the clover cover crop and the black plastic treatments significantly impacted weed

18 biomass compared to the control, there were no significant differences in means for most tomato

19 crop vitality measures during both the first and second data collection periods. Given that crop

20 yield is a key parameter driving agricultural decisions, it is particularly important to note the lack

21 of significant difference in means of the number of fruit per plant between the treatments for the

22 tomatoes overall during the first $(\mathrm{p}=0.6891)$, second $(\mathrm{p}=0.8005)$, and third data collection

23 periods $(p=0.6709)$. Significant differences in means were seen between the three treatments $(p$ 
$1<0.0021)$ during the second data collection for leaf color intensity, a proxy for photosynthetic

2 potential and plant health, with the clover cover cropping treatment having the highest leaf color

3 intensity (score of 3.76 on a $0-5$ scale) followed by the black plastic treatment (3.12), and then

4 the control (2.96). In addition, significant differences in means were seen during the second data

5 collection for the overall tomatoes for the number of branching stems between the three

6 treatments $(\mathrm{p}<0.0102)$ with the clover cover cropping treatment having the greatest number of

7 branching stems (mean of 4.84) followed by the black plastic treatment (3.88), and then the

8 control (3.68).

9 Soil Quality. Significant differences were found in the means of several soil parameters

10 across the three weed suppression strategies for the overall tomato experiment. For soil nutrients,

11 significant differences in means were found for phosphorus concentrations $(\mathrm{p}<0.0003)$ and

12 potassium concentrations $(\mathrm{p}<0.0001)$ while no significant differences were seen in nitrogen

13 concentrations ( $\mathrm{p}>$ across the weed treatments. The clover cover cropping treatment had

14 sufficient phosphorus levels for tomatoes while the control had adequate levels and the black

15 plastic treatment had deficient levels. The clover cover cropping treatment and the control had

16 significantly higher levels of phosphorus compared to the black plastic treatment. Additionally,

17 the clover cover cropping treatment and the control both had sufficient levels of potassium that

18 were significantly higher than the black plastic treatment that had adequate levels of potassium.

19 For soil moisture content, significant differences in means were found across the three treatments

$20(\mathrm{p}<0.0001)$ with the black plastic treatment having significantly higher moisture levels

21 compared to control and the clover cover cropping treatment. Significant differences were

22 further observed between the weed suppression treatments for soil temperature at $15 \mathrm{~cm}$ below 
1 ground $(\mathrm{p}<0.0039)$ with the black plastic having significantly higher temperature than the

2 clover cover cropping treatment.

3 Crop Quality. Significant differences were found in means of overall sensory desirability

4 of tomato fruit across the three treatments $(\mathrm{p}<0.01)$ with the black plastic treatment having

5 significantly higher desirability (score of 3 on a scale of 0-5) compared to the clover cover

6 treatment (score of 2.5) and the control (score of 2.5; Figure S5). For total phenolic concentration

7 (TPC; a phytochemical measure for health-promoting attributes, flavor, appearance, and shelf-

8 life), significant differences were found across all three weed treatments with $(\mathrm{p}<0.0001$; Figure

9 S6) with the clover cover cropping treatment having the highest TPC values (7.01 $\mathrm{mg} \mathrm{GAE} \mathrm{/} \mathrm{g)}$

10 followed by the control (5.53 $\mathrm{mg} \mathrm{GAE} / \mathrm{g})$ and the black plastic treatment ( $4.28 \mathrm{mg} \mathrm{GAE} / \mathrm{g})$. No

11 significant differences were found in the means brix levels across the three weed suppression

12 strategies for the overall tomato experiment.

\section{Discussion}

14 This study highlights the multiple effects that weed suppression strategies have on tomato

15 cropping systems and the importance of measuring multiple outcomes to understand the multiple

16 variables that farm managers are presented with in their decision making. In addition, findings

17 emphasize how different cultivars respond differently for different outcomes and the importance

18 of making sustainability decisions on a context specific basis that account for such diversity.

19 Both clover cover crop and black plastic had significant effects in reducing weeds in the tomato

20 plots compared to the control treatment. The abundance of specific weeds varied between the

21 three treatments indicating different properties of the soil. The variation in weed prevalence

22 directly influences time and cost of farm labor for weeding. The clover cover cropping treatment

23 contributed to overall farm diversity at the Dartmouth Organic Farm. These findings highlight 
1 the various outcomes and tradeoffs that farmers are faced with in making agricultural

2 management decisions.

3 While the clover cover crop and black plastic treatments were effective in suppressing

4 weeds, neither of these treatments resulted in a significant difference in the number of fruit,

5 which is the key output for harvest and sales that drive farmers' decision making. However, the

6 black plastic treatment did have significantly higher means for several plant vitality and quality

7 measures as well as soil properties while the clover cover crop treatment had significantly higher

8 means for other plant vitality and quality measures. For example, the tomatoes of the black

9 plastic treatment were rated as overall more desirable in the sensory evaluation compared to the

10 other treatments indicating its greater consumer preference and thus attractiveness for producers

11 to cultivate to ensure a desirable product. The black plastic treatment was also found to be most

12 effective in water conservation through retaining soil moisture retention and insulating the soil,

13 which are beneficial properties for maintaining soil moisture during summer months as well as

14 for optimal temperatures for tomato plants during cooler New Hampshire nights. From a labor

15 and economics perspective, the black plastic treatment required the lowest labor input and thus

16 was most economically feasible based on cost of inputs. At the same time, the tomatoes of the

17 clover cover cropping treatment had the highest total phenolic concentration which is a

18 phytochemical measure for health-promoting attributes, flavor, appearance, and shelf-life.

19 However, consumers may not value the increased phenolic concentration based on flavor as

20 indicated by the lower sensory desirability scores of the tomatoes from this treatment.

21 Given that there were no significant differences in the means for the abundance of weeds

22 and the number of fruit between the clover cover crop and the black plastic treatment, students of

23 ENVS 25 suggested that cover cropping should be used for weed suppression at the Dartmouth 
1 Organic Farm. As a petroleum-based product that is difficult to recycle and ultimately goes to

2 landfills, the black plastic mulch is recognized as inherently unsustainable. However, students of

3 ENVS 25 recognized the additional labor inputs required for weeding under the cover cropping

4 management system compared to the plastic mulch system. At the same time, students

5 recognized that the clover cover cropping treatment contributed to overall farm diversity at the

6 Dartmouth Organic Farm and suggested this as one of the management strategies to increase on-

7 farm diversity (Figure S7). In a trade-off between labor input and biodiversity at the scale of the

8 Dartmouth Organic Farm, students of ENVS 25 supported that the cover cropping practice for

9 weed management was feasible. Students suggested that small-holder farm managers who

10 currently use black plastic should consider using alternative weed suppression methods.

11 However, students recognized the limitations of scaling this practice to larger farms given

12 constraints of labor. Students further reflected on the potential social justice issues related to

13 making agricultural decisions with regards to needs for labor input.

14 For future FARMS experiments for ENVS 25, students suggested to compare black

15 plastic mulch to biodegradable mulches and measure additional outcomes in tomato cropping

16 systems such as the effects on microbial communities as well as the influence of weed

17 suppression strategies on soil temperatures with projected climate change scenarios. As the cover

18 crop treatment positively impacted a few plant vitality measures, students suggested further

19 research regarding cover cropping including comparisons with other types of cover crops and

20 comparisons between diverse mixtures of cover crop species in order to obtain multiple benefits

21 associated with agricultural diversification.

\section{References}


1 Ahmed, S, Unachukwu, U, Stepp, JR, Peters, CM, -Chunlin, L and Kennelly, E 2010 Pu-erh tea

2 tasting in Yunnan, China: Correlation of drinkers' perceptions to phytochemistry. J

3 Ethnopharmacol 132: 176-185. DOI: https://doi.org/10.1016/j.jep.2010.08.016

4 Liebman, M and Gallandt, ER 1997 Many little hammers: Ecological management of crop-weed

5 interactions. In: Jackson, LE (ed.), Ecology in agriculture, 291-343. Academic Press, New York.

6 DOI: https://doi.org/10.1016/B978-012378260-1/50010-5

7 Pfeiffer, EE 1974 Weeds and what they tell, 96. Springfield: Bio-dynamic Farming and

8 Gardening Association.

9 Rodale's Organic Life 2012 Listen to your weeds! Put your ear to the ground and hear what your

10 weeds are saying about your soil. https://www.rodalesorganiclife.com/garden/listen-your-weeds.

11 United States Department of Agriculture (USDA) 2012 USDA plant hardiness zone map.

12 Available at: http://www.planthardiness.ars.usda.gov/. Accessed August 25, 2016.

13 